\title{
Cheating death: a Coxiella effector prevents apoptosis
}

\section{Laura J. Broederdorf and Daniel E. Voth*}

Department of Microbiology and Immunology, University of Arkansas for Medical Sciences, Little Rock, AR, USA

*Correspondence: dvoth@uams.edu

\section{A commentary on}

Inhibition of pathogen-induced apoptosis by a Coxiella burnetii type IV effector protein

by Luhrmann, A., Nogueira, C. V., Carey, K. L., and Roy, C. R. (2010). Proc. Natl. Acad. Sci. U.S.A. 107, 18997-19001.

Intracellular bacterial pathogens have developed strategies to subvert numerous host cell processes, often by deploying a battery of secreted proteins, termed effectors, into the cytosol. The stealthy agent of Q fever, Coxiella burnetii, continuously manipulates its eukaryotic host cell throughout a prolonged infectious cycle and replicates in a unique phagolysosome-like vacuole. The organism encodes a Dot/Icm type IV secretion system (T4SS) similar to that of closely related Legionella pneumophila, the causative agent of Legionnaires' disease (Seshadri et al., 2003). These pathogens' respective T4SSs are predicted to translocate a large number of effectors directly from the vacuole into the host cell cytosol where they interact with eukaryotic proteins to influence infection events. While over 300 Legionella Dot/Icm substrates have been identified (Hubber and Roy, 2010), Coxiella encodes few obvious homologs of these or other bacterial effectors, indicating the use of pathogen-specific repertoires. However, T4SS conservation allows the use of Legionella to study Coxiella effectors, expanding the panel of tools available for this genetically intractable organism.

Recent studies show Coxiella isolates encode 14 Ank proteins containing eukaryotic-like ankyrin repeat domains, 11 of which are translocated by the Legionella Dot/Icm T4SS (Pan et al., 2008; Voth et al., 2009). However, Ank function has remained a mystery until a recent report by Luhrmann et al. (2010) examining Coxiella effectordriven anti-apoptotic activity. Coxiella antagonizes intrinsic apoptotic death in macrophages (Voth et al., 2007) and prevents cytochrome $c$ release from mitochon- dria to provide a stable intracellular niche for replication (Luhrmann and Roy, 2007). The pathogen also activates Akt and Erk1/2 signaling to promote survival (Voth and Heinzen, 2009). Each of these events relies on Coxiella protein synthesis, suggesting the organism secretes a distinct effector(s) to regulate apoptosis. Unfortunately, effector identification and characterization has been hampered by a lack of methods for Coxiella genetic manipulation. As an alternative, Luhrmann et al. (2010) investigated the mechanistic activity of a Coxiella Ank through exploration of its anti-apoptotic function in Legionella.

Luhrmann et al. (2010) tested the ability of four Anks (AnkA, B, F, and G) to inhibit intrinsic apoptosis. Eukaryotic cells ectopically expressing individual Anks were treated with the apoptosis-inducing agent staurosporine. Only AnkG prevented apoptosis with $\sim 65 \%$ of AnkG-expressing cells maintaining viability. Ankyrin repeat domains mediate eukaryotic proteinprotein interactions (Mosavi et al., 2004) and Dot/Icm substrates predictably bind to, and manipulate, a specific host protein(s). Therefore, Luhrmann et al. (2010) used GST pulldown and immunoprecipitation approaches to identify host-binding partners for AnkG. Interestingly, mass spectrometry analysis and confirmatory immunoprecipitation studies identified the mitochondrial inner matrix protein p32 as a specific AnkG interacting protein. p32 is a pro-apoptotic protein that binds to Hrk to promote cytochrome $c$ release (Sunayama et al., 2004) and also interacts with ARF, a pro-apoptotic p53 regulatory protein (Itahana and Zhang, 2008). Thus, AnkG interaction with p32 likely precludes interaction with other pro-apoptotic mitochondrial proteins, resulting in decreased cytochrome $c$ release. This is also the first known example of a bacterial protein targeting p32 to manipulate host cell survival.

Next, the authors performed a set of interesting gain of function experiments. In contrast to Coxiella, Legionella induces rapid apoptosis in some cell types, such as dendritic cells (DCs; Nogueira et al., 2009). Therefore, the authors hypothesized that Coxiella AnkG would provide Legionella with a tool to inhibit DC apoptosis and allow replication. Mouse bone marrow-derived DCs were infected with Legionella expressing Coxiella AnkG, then assessed for apoptosis. Remarkably, adding AnkG to Legionalla's effector repertoire reduced DC apoptosis by $\sim 40 \%$. Infecting cells with Legionella producing truncated AnkG showed the p32-interacting region was required for inhibition of apoptosis, highlighting the functional importance of an effector binding to a host protein. Additionally, siRNA-mediated dampening of p32 expression reduced Legionellatriggered DC apoptosis similar to AnkG production. Collectively, the experiments performed by Luhrmann et al. (2010) provide a mechanism of AnkG anti-apoptotic activity and demonstrate the use one pathogen's effector to study another organism's intracellular activity.

Luhrmann et al. (2010) provide the first glimpse into how a Coxiella effector interacts with a host protein to alter a distinct infection event (Figure 1). This study also further underscores the differences between Coxiella and Legionella. Despite similar T4SSs, the effector repertoires of these two pathogens are highly divergent yet required for each organism's intracellular lifestyle. The intriguing results of Luhrmann et al. (2010) also foster some remaining questions about the mechanism of AnkG-mediated protection. First, does translocated AnkG traffic to mitochondria to initiate p32 interactions? AnkG could potentially bind to cytosolic p32, preventing proper localization and interaction with mitochondrial proteins. Second, is AnkG binding to p32 alone sufficient to inhibit cytochrome $c$ release? Third, does AnkG influence death receptor-mediated extrinsic apoptosis? Finally, do other Anks contribute to AnkG activity? In their initial screen, the authors showed that AnkF pro- 
vides modest protection from apoptosis. Perhaps AnkF works in concert with AnkG to fully protect host cells from death. This prediction is not unprecedented, as four Legionella anti-apoptotic effectors target different host proteins (Banga et al., 2007; Ge et al., 2009). Luhrmann et al. (2010) have defined an important role for AnkG in Coxiella-host cell interactions and demonstrated elegant experimental approaches that will serve as a blueprint for functional characterization of other Coxiella Dot/Icm substrates. It will also be interesting to see if Legionella can be exploited to study Coxiella effectors that direct other processes such as phagolysosome formation.

\section{REFERENCES}

Banga, S., Gao, P., Shen, X., Fiscus, V., Zong, W. X., Chen, L., and Luo, Z. Q. (2007). Legionella pneumophila inhibits macrophage apoptosis by targeting pro-death members of the Bcl 2 protein family. Proc. Natl. Acad. Sci. U.S.A. 104, 5121-5126.

Ge, J., Xu, H., Li, T., Zhou, Y., Zhang, Z., Li, S., Liu, L., and Shao, F. (2009). A Legionella type IV effector activates the NF-kappaB pathway by phosphorylating the IkappaB family of inhibitors. Proc. Natl. Acad. Sci. U.S.A. 106, 13725-13730.

Hubber,A., and Roy, C. R. (2010). Modulation of host cell function by Legionella pneumophila type IV effectors. Annu. Rev. Cell Dev. Biol. 26, 261-283.

Itahana, K., and Zhang, Y. (2008). Mitochondrial p32 is a critical mediator of ARF-induced apoptosis. Cancer Cell 13, 542-553.

Luhrmann, A., Nogueira, C. V., Carey, K. L., and Roy, C. R. (2010). Inhibition of pathogen-induced apoptosis by a Coxiella burnetii type IV effector protein. Proc. Natl. Acad. Sci. U.S.A. 107, 18997-19001.

Luhrmann,A., and Roy, C.R. (2007). Coxiellaburnetii inhibits activation of host cell apoptosis through a mechanism that involves preventing cytochrome c release from mitochondria. Infect. Immun. 75, 5282-5289.

Mosavi, L. K., Cammett, T. J., Desrosiers, D. C., and Peng, Z. Y. (2004). The ankyrin repeat as molecular architecture for protein recognition. Protein Sci. 13, 1435-1448.

Nogueira, C. V., Lindsten, T., Jamieson, A. M., Case, C. L., Shin, S., Thompson, C. B., and Roy, C. R. (2009). Rapid

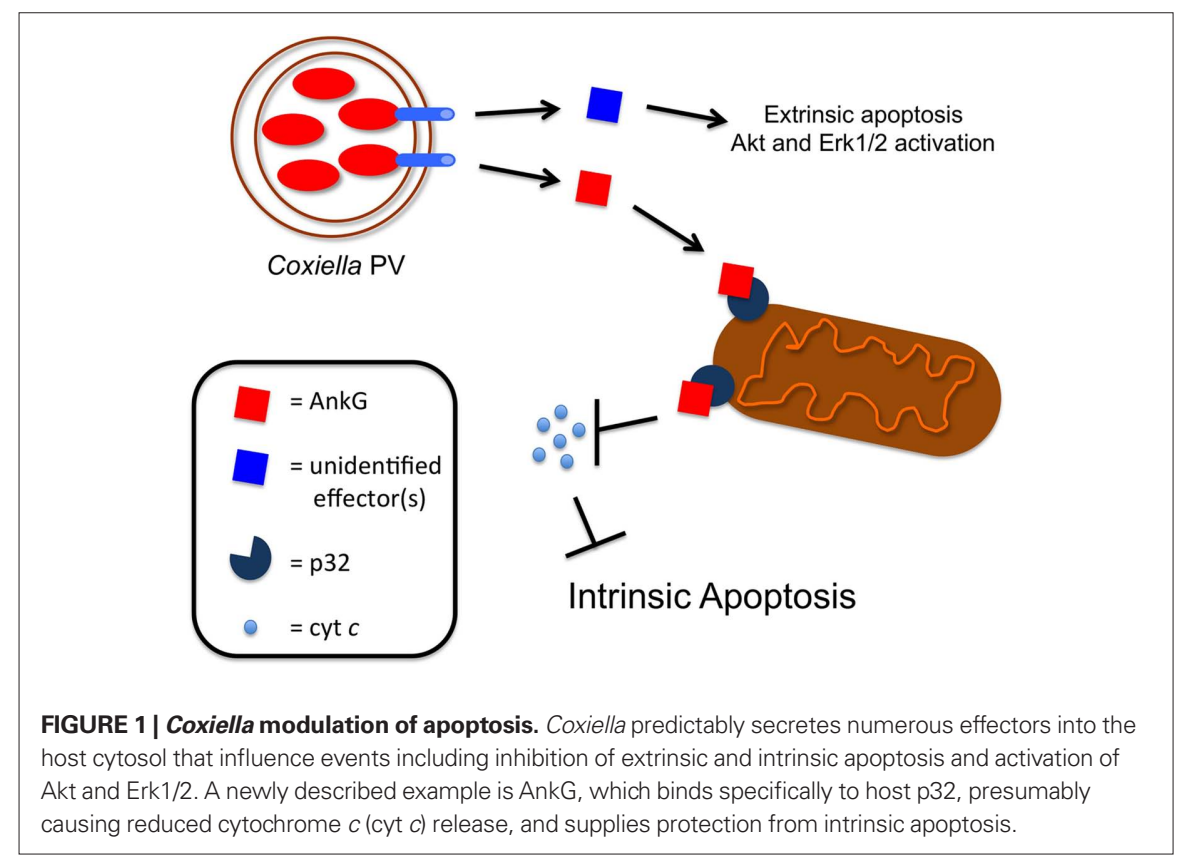

pathogen-induced apoptosis: a mechanism used by dendritic cells to limit intracellular replication of Legionella pneumophila. PLoS Pathog. 5, e1000478. doi: 10.1371/journal.ppat.1000478

Pan, X., Luhrmann, A., Satoh, A., Laskowski-Arce, M. A., and Roy, C. R. (2008). Ankyrin repeat proteins comprise a diverse family of bacterial type IV effectors. Science 320, 1651-1654.

Seshadri, R., Paulsen, I. T., Eisen, J. A., Read, T. D., Nelson, K. E., Nelson, W. C., Ward, N. L., Tettelin, H., Davidsen, T. M., Beanan, M. J., Deboy, R. T., Daugherty, S. C., Brinkac, L. M., Madupu, R., Dodson, R. J., Khouri, H. M., Lee, K. H., Carty, H. A., Scanlan, D., Heinzen, R. A., Thompson, H. A., Samuel, J. E., Fraser, C. M., and Heidelberg, J. F. (2003). Complete genome sequence of the Q-fever pathogen Coxiella burnetii. Proc. Natl. Acad. Sci. U.S.A. 100, 5455-5460.

Sunayama, J., Ando, Y., Itoh, N., Tomiyama, A., Sakurada, K., Sugiyama, A., Kang, D., Tashiro, F., Gotoh, Y., Kuchino, Y., and Kitanaka, C. (2004). Physical and functional interaction between $\mathrm{BH}$-only protein $\mathrm{Hrk}$ and mitochondrial pore-forming protein p32. Cell Death Differ. 11, 771-781.

Voth, D. E., and Heinzen, R. A. (2009). Sustained activation of Akt and Erk1/2 is required for Coxiella burnetii antiapoptotic activity. Infect. Immun. 77, 205-213.

Voth, D. E., Howe, D., Beare, P. A., Vogel, J. P., Unsworth, N., Samuel, J. E., and Heinzen, R. A. (2009). The Coxiella burnetii ankyrin repeat domain-containing protein family is heterogeneous, with C-terminal truncations that influence Dot/Icm-mediated secretion. J. Bacteriol. 191, 4232-4242.

Voth, D. E., Howe, D., and Heinzen, R. A. (2007). Coxiella burnetii inhibits apoptosis in human THP-1 cells and monkey primary alveolar macrophages. Infect. Immun. 75, 4263-4271.

Received: 21 February 2011; accepted: 23 February 2011; published online: 07 March 2011.

Citation: Broederdorf LJ and Voth DE (2011) Cheating death: a Coxiella effector prevents apoptosis. Front.

This article was submitted to Frontiers in Cellular and Infection Microbiology, a specialty of Frontiers in Microbiology.

Copyright (C) 2011 Broederdorf and Voth. This is an openaccess article subject to an exclusive license agreement between the authors and Frontiers Media SA, which permits unrestricted use, distribution, and reproduction in any medium, provided the original authors and source are credited. Microbio. 2:43. doi: 10.3389/fmicb.2011.00043 